\title{
Dermatopontin inhibits WNT signaling pathway via CXXC finger protein 4 in hepatocellular carcinoma
}

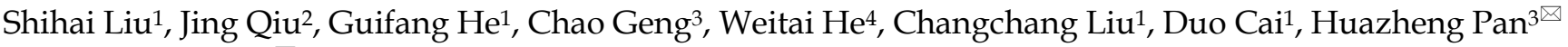 \\ and Qingwu Tian ${ }^{3}$ \\ 1. Medical Animal Lab, The Affiliated Hospital of Qingdao University, Qingdao, 266000, China. \\ 2. Department of Stomatology, Qingdao Municipal Hospital, Qingdao, 266071, China. \\ 3. Department of Clinical Laboratory, The Affiliated Hospital of Qingdao University, Qingdao, 266000, China. \\ 4. School of Biological Science and Technology, University of Jinan, Jinan, 250022, China. \\ $\triangle$ Corresponding authors: Huazheng Pan, Ph.D. Department of Clinical Laboratory, The Affiliated Hospital of Qingdao University, No. 16, Jiangsu Road, \\ Qingdao 266000, P.R. China. Tel.: +86 532 82911773; Fax: +86 532 82911773; E-mail: panhuazheng@126.com; Qingwu Tian, Professor, Department of Clinical \\ Laboratory, The Affiliated Hospital of Qingdao University, No. 1677, Wutaishan Road, Qingdao 266000, P.R. China. Tel.: +86 53282919388 ; Fax: +86 532 \\ 82919388; E-mail: tianqingwu1970@163.com.
}

(c) The author(s). This is an open access article distributed under the terms of the Creative Commons Attribution License (https://creativecommons.org/licenses/by/4.0/). See http://ivyspring.com/terms for full terms and conditions.

Received: 2020.04.18; Accepted: 2020.08.10; Published: 2020.08.29

\begin{abstract}
Hepatocellular carcinoma $(\mathrm{HCC})$ is a major cause of tumor associated deaths globally. Annually, the prevalence of HCC is increasing and the lack of early prognostic indicators manifests a dismal prognosis for HCC patients. A deep understanding of the molecular events that promote HCC progression are required for the design of new diagnostics and therapeutics. Dermatopontin (DPT) is an extracellular matrix protein that regulates the metastatic phenotypes of many cancers. However, the effects of DPT on $\mathrm{HCC}$ cell growth remain undefined. In this study, we demonstrate that the exogenous expression of DPT inhibits HCC cell growth both in vitro and in vivo. Furthermore, we show that DPT regulates CXXC4, which in turn targets c-Myc, EZH2, SOX2 and $\beta$-catenin, through its ability to impact Wnt signaling pathway. These data suggest that DPT regulates CXXC4, c-Myc, EZH2, SOX2 and $\beta$-catenin, through Wnt signaling to repress HCC proliferation. This highlights DPT as promising target for future HCC diagnostics and therapeutic targets.
\end{abstract}

Key words: Dermatopontin, Wnt pathway, Hepatocellular carcinoma

\section{Introduction}

Hepatocellular carcinoma (HCC) is a leading cause of liver cancer globally and the $2^{\text {nd }}$ highest cause of cancer associated death in East Asia [1]. Current HCC diagnostics are ineffective during the early disease stages, leading to many patients diagnosed with late stage disease leading to a poor prognosis. Identifying new diagnostic and therapeutic targets to improve HCC treatment are urgently required.

Purified from the bovine dermis, dermatopontin $(\mathrm{DPT})$ is an extracellular matrix $(\mathrm{ECM})$ protein rich in tyrosine residues [2]. DPT influences the ECM through its ability to enhance fibronectin and collagen fibrillogenesis and through its interactions with decorin and TGF- $\beta$, ultimately leading to increase the biological activity of TGF- $\beta[3,4]$. Due to its roles in cell-to-cell adhesion and ECM development, DPT regulates multiple physiological processes. The inhibition of DPT is associated with a range of pathologies including systemic sclerosis, uterine leiomyomas, cutaneous fibrosis, and several cancers [5]. DPT also inhibits the metastatic properties of oral squamous cell carcinoma (OSCC), though the mechanism(s) of these effects have not been elucidated [6]. However, the biological effects of DPT during HCC development have not been defined.

In the current study, we demonstrate that DPT is downregulated in clinical HCC samples and that the exogenous overexpression of DPT inhibits HCC cell proliferation. We further demonstrate that DPT regulates $\mathrm{CXXC4}$, which in turn targets the c-Myc, EZH2 and SOX2 axis through its ability to regulate 
Wnt/ $\beta$-catenin signaling. These data highlight the suppression of DPT as an important driver of HCC development, and furthers our understanding of HCC development and future avenues of therapeutic strategies.

\section{Materials and Methods}

\section{Patient tissue}

HCC tissues and paired adjacent non-HCC tissues ( $\mathrm{n}=60$ for both) were obtained for patients in our hospital. Informed consent was provided for tissue collection and all protocols were approved by our ethics committee. Patients received surgery with no adjuvant radiotherapy or chemotherapy. Collected tissues were frozen at $-80^{\circ} \mathrm{C}$ prior to analysis. HCC was confirmed in all isolated patient materials independently by two experienced pathologists. The study methodologies conformed to the standards set by the Declaration of Helsinki.

\section{Cell lines and transfection}

HL7702 cells (non-HCC) and Huh7, Hep3B, HCCLM3 and PLC/PRF/5 cells (liver cancer cell lines) were maintained under standard cell culture conditions [7]. Briefly, cells were grown in EMEM or DMEM plus 10\% Fetal Bovine Serum (FBS), penicillin/streptomycin, and glutamine $(2 \mathrm{mM})$. For DPT overexpression studies, p3 $\times$ Flag-DPT was purchased from Geneppl technologies (Nanjing, China). For CXXC4 overexpression studies GV141-CXXC4-3XFlag was purchased from GeneChem (Shanghai, China). Silencing studies were performed with shRNAs targeting DPT (shDPT), CXXC4 (shCXXC4), or scrambled controls (shCON). All shRNAs were purchased from GenePharma (Shanghai, China). Transfections were performed using Lipofectamine 3000 (Invitrogen, Carlsbad, CA) as the manufacturer's recommendations.

\section{3-(4,5-dimethylthiazol-2-yl)-2,5-diphenyltetraz olium bromide (MTT) assays}

For cell viability assessments, HCC cells $(\sim 10,000$ per well of a 96 well plate) were transfected for 24,48 and $72 \mathrm{~h}$ and subsequently treated with MTT reagent (Sigma-Aldrich, St. Louis, MO) for $2 \mathrm{~h}$. Formazan produced by the cells was then solubilized in DMSO (Sigma-Aldrich, St. Louis, MO). MTT absorbances were measured at $450 \mathrm{~nm}$ on a 96-well microplate reader (Bio-Rad, Hercules, CA). All values were normalized to control cells for viability assessments.

\section{5-Ethynyl-2'-deoxyuridine (EdU) proliferation assays}

EdU incorporation assays were used to measure HCC cell proliferation. Briefly, HCC cells $\left(1 \times 10^{5}\right.$ cells/well) were grown in dishes and treated for $48 \mathrm{~h}$. Cells were then labeled with $50 \mu \mathrm{M}$ EdU (Beyotime Institute of Biotechnology, Shanghai, China) for $2 \mathrm{~h}$ at $37^{\circ} \mathrm{C}$. Cells were subsequently fixed in $4 \%$ PFA for 30 min, permeabilized with $0.5 \%$ Triton X-100 for $30 \mathrm{~min}$ and incubated with Apollo reaction cocktail (500 $\mu \mathrm{L} /$ well) for $30 \mathrm{~min}$. Cell nuclei were Hoechst 33342 (Beyotime Institute of Biotechnology, Shanghai, China) stained and cells were imaged via fluorescence microscopy (10 fields of view per treatment). EdU-positive cells were counted on a Nikon, 80i microscope (Nikon, Japan). Average EdU intensities per cell were measured.

\section{Preparation of cell extracts and Western blotting}

Cells were harvested in RIPA buffer plus protease/phosphatase inhibitors and protein content were quantified via Bio-Rad assays. In total, 20-50 $\mu \mathrm{g}$ of each sample were resolved by SDS-PAGE and proteins were semi-dry transferred onto PVDF membranes (Millipore Corp., Bedford, MA) and blocked for $1 \mathrm{~h}$ in Blotto A (5\% milk in TBS-T), followed by labeling for $1 \mathrm{~h}$ with Blotto A containing anti-DPT (Cat. ab255823, Abcam, Cambridge, MA), anti-EZH2 (Cat. ab191250, Abcam, Cambridge, MA), anti- $\beta$-catenin (Cat. \#8480, CST, Danvers, MA), antiSOX2 (Cat. \#4900, CST, Danvers, MA), c-Myc (Cat. ab39688, Abcam, Cambridge, MA), anti-p-GSK3 $\beta$ (Cat. \#5558, CST, Danvers, MA), CXXC4 (Cat. ab105400, Abcam, Cambridge, MA) and anti-GAPDH (Cat. ab8245, Abcam, Cambridge, MA) antibodies. Blots were washed and labeled in Blotto A containing the appropriate HRP-conjugated secondary antibodies (CST, Danvers, MA). Membranes were washed in TBS-T. The ECL system was used for band visualization and values were quantified and normalized to GAPDH as a housekeeper control.

\section{Luciferase reporter assays}

The Promega dual luciferase system was used for TOPFlash/FOPFlash reporter assays (Promega, Madison, WI). Briefly, cells were co-transfected with TOPFlash/FOPFlash for $24 \mathrm{~h}$ and lysed in commercially available Dual-Luciferase Reporter Assay buffer. Firefly and Renilla activity were then assessed on a luminometer. Data presented is firefly luciferase activity after normalization with Renilla luciferase.

\section{Quantitative real time Polymerase Chain Reaction (qPCR) analysis}

RNA was purified using commercial RNeasy Mini kits (Qiagen, Hilden, Germany) according to the manufacturer's recommendations. CDNA synthesis 
kits (iScript, Bio-Rad, Hercules, CA) were used for first stand synthesis. Gene expression profiles of Wnt related genes $(n=84)$ were assessed using the Wnt Signaling Pathway $\mathrm{RT}^{2}$ Profiler PCR Arrays (Cat. PAHS-043ZR, Qiagen) on a 7500 Sequence Detection System (Applied Biosystems, Foster City, CA) plus recommended controls. Relative gene expression was assessed using a Qiagen RT ${ }^{2}$ Profiler PCR Array via the CT method. Values were normalized to GAPDH as a reference gene. PCR arrays, whose threshold cycle above the 35th cycle, were excluded. Data were analyzed using Qiagen web-based data analysis.

\section{Clonogenic assays by soft agar}

To assess the colony formation capacity of HCC cells, cells were harvested in trypsin and seeded $(2 \times$ $10^{3}$ cells per 6-well plate) in complete DMEM containing $0.3 \%$ and $0.6 \%$ agar. Cells were then maintained under standard culture conditions for a 15 days period, at which time, colonies were fixed in $70 \%$ ethanol and imaged. Colonies $\geq 50$ cells were deemed positive.

\section{Cell imaging by immunofluorescence (IF)}

For IF analysis, cells were fixed in 4\% PFA, treated with pepsin, and dehydrated using a gradient ethanol series. Cells were then permeabilized in Triton X-100, blocked in PBS plus goat serum, and probed with anti- $\beta$-catenin antibodies (Cat. \#8480, CST, Danvers, MA) overnight. Cells were washed and labeled with the appropriate fluorescently conjugated secondary antibodies. Nuclei were counter-stained with 4',6-diamidino-2-phenylindole (DAPI) and imaged on a Leica TCS SPE confocal and DM 5500 Q microscope (Leica Microsystems, Germany). For all data, representative confocal images were shown.

\section{Co-Immunoprecipitation (Co-IP)}

Cells $\left(2 \times 10^{6}\right)$ were lysed in Co-IP buffer containing Tris- $\mathrm{HCl}(\mathrm{pH} 7.5), 150 \mathrm{mM} \mathrm{NaCl}, 1 \%$ Nonidet P-40, and 10\% glycerol plus protease/ phosphatase inhibitors. Lysates were pre-cleared and labeled with anti-flag antibodies (Cat. \#14793, CST, Danvers, MA) or anti-Dvl-1 (Cat. sc-8025, Santa Cruz, CA) overnight at $4^{\circ} \mathrm{C}$ with mixing. Protein A/G conjugated to agarose (Santa Cruz, CA) was then added for $2 \mathrm{~h}$ and pelleted through centrifugation. Control samples contained rabbit IgG alone. Beads were washed in IP buffer ( $\times 3$ times) and mixed with 2 $\times$ loading buffer. Samples were boiled for $5 \mathrm{~min}$ and assessed by Western blotting analysis [8]. Representative Co-IPs are shown throughout.

\section{Tumor assessments}

Tumor growth assays were performed in athymic BalB/C nude mice (obtained from the Beijing
Vital River Laboratory). HCCLM3 cells $\left(1 \times 10^{6}\right)$ were injected into the left dorsal flank ( $\mathrm{n}=6$ per treatment) and monitored daily. Upon tumor sizes of 40 to 50 $\mathrm{mm}^{3}$ being observed, mice were treated for 5 days. An electronic digital caliper was used to assess tumor growth characteristics and tumor volumes were calculated using the equation: tumor size $=a \times b^{2} / 2, a$ : larger and $b$ : smaller of the dimensions. Mice were sacrificed $24 \mathrm{~d}$ post-injection and tumor characteristics were examined.

\section{Statistics}

All statistical analyses were carried out using SPSS version 18.0 (IBM Corp., Armonk, NY). The associations between DPT expression and the clinicopathological characteristics of the patients were analyzed using the Chi-squared test. Bivariate correlations between study variables were calculated using the Spearman's rank correlation coefficient. Survival curves were plotted using the Kaplan-Meier method and compared using the log-rank test. Data are shown as the mean \pm SD. $P<0.05$ indicated a significant difference between treatment groups.

\section{Results}

\section{DPT is downregulated in HCC tissues and correlates with patient survival}

A deep understanding of the molecular events that promote HCC progression are required for the effective development of new therapeutics. DPT is an extracellular matrix protein that regulates the metastatic phenotypes an array of human neoplasms. We initially assessed the expression of DPT in Cancer transcription analysis on TCGA samples from UALCAN database (http://ualcan.path.uab.edu/ index.html) and GEPIA database (http://gepia. cancer-pku.cn/). UALCAN showed that DPT was downregulated expressed in HCC compared with that in normal liver tissues (Figure S1A). We got the similar results in GEPIA database (Figure S1B). DPT expression was positively correlated with the clinical stage and pathological grade (Figure S1C and S1D). Then, we assessed DPT expression in 60 paired HCC tissues obtained from patients in our hospital who underwent surgery. HCC was confirmed in all samples by an experienced panel of pathologists. From IHC analysis, DPT had a cytoplasmic distribution (Figure 1A) indicative of a soluble protein. Western blotting analysis further showed that the total levels of DPT expression in surgical tissues from non-cancerous $(\mathrm{N})$ were higher than that of HCC tumor tissue (T) (Figure 1B). To investigate the frequency of DPT regulation in HCC, we examined the expression using qPCR in 60 fresh-frozen HCC 
tissues, including 52 cases at clinical stage I and II, 8 cases at clinical stage III and IV (Table 1). We observed significant downregulation of DPT in HCC tissues (Figure 1C). Then we analyzed the association between DPT and the clinicopathological features of HCC. As shown in Table 2, strong associations were observed between DPT expression and age $(P=0.048)$ and alcoholism $(P=0.029)$. However, the expression of DPT was not associated with gender $(P=0.648)$, liver cirrhosis $(P=0.570)$, AFP level $(P=0.855)$, ALT $(P=0.128)$, AST $(P=0.144)$, tumor number $(P=0.206)$, tumor size $(P=0.993)$, portal vein invasion $(P=0.282)$ or TNM stage $(P=0.156)$. Spearman analysis of correlation between DPT and clinicopathological features revealed that the expression of DPT was significantly correlated with age $(P=0.049)$ and alcoholism $(P=0.029)$ (Table 3$)$. Kaplan-Meier survival curves demonstrated that the overall survival of patients with high expression of DPT was significantly longer than those with low DPT expression (Figure 1D, $P=0.0491$ ). Collectively, these results indicate that downregulation of DPT in primary HCC patients correlate with poor survival.

Table 1. Clinicopathological characteristics of patient samples and expression of DPT in HCC

\begin{tabular}{ll}
\hline Characteristics & No. of case $(\%)$ \\
\hline Age & $14(23.3)$ \\
$<50$ & $46(76.7)$ \\
$\geq 50$ & $49(81.7)$ \\
Gender & $11(18.3)$ \\
Male & \\
Female & $16(26.7)$ \\
Alcoholism & $44(73.3)$ \\
Yes & \\
No & $35(58.3)$ \\
Liver cirrhosis & $25(41.7)$ \\
Yes & \\
No & $40(66.7)$ \\
AFP (ng/L) & $20(33.3)$ \\
$<200$ & $42(70.0)$ \\
$\geq 200$ & $30(30.0)$ \\
ALT (U/L) & \\
$<60$ & $40(66.7)$ \\
$\geq 60$ & $20(33.3)$ \\
AST (U/L) & \\
$<40$ & $36(60.0)$ \\
$\geq 40$ & $24(40.0)$ \\
Tumor number & \\
Single & $31(51.7)$ \\
Multiple & $29(48.3)$ \\
Tumor size & \\
$<5 \mathrm{~cm}$ & $13(21.7)$ \\
$\geq 5 \mathrm{~cm}$ & $47(78.3)$ \\
Portal vein invasion & $52(86.7)$ \\
Yes & $8(13.3)$ \\
No & TNM stage \\
I+II stage & \\
III+IV stage & \\
\hline &
\end{tabular}

HCC, hepatocellular carcinoma; AFP, a-fetoprotein; ALT, alanine aminotransferase; AST, aspartate aminotransferase; TNM, tumor node metastasis.
Table 2. Correlation between DPT expression and clinicopathologic characteristics of $\mathrm{HCC}$ patients

\begin{tabular}{|c|c|c|c|}
\hline \multirow[t]{2}{*}{ Characteristics DPT } & \multicolumn{3}{|l|}{ DPT expression } \\
\hline & Low or none, no. cases & High, no. cases & $p$ value \\
\hline \multicolumn{4}{|c|}{ 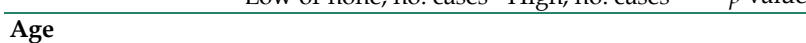 } \\
\hline$<50$ & 4 & 10 & 0.048 \\
\hline$\geq 50$ & 27 & 19 & \\
\hline \multicolumn{4}{|l|}{ Gender } \\
\hline Male & 26 & 23 & 0.648 \\
\hline Female & 5 & 6 & \\
\hline \multicolumn{4}{|l|}{ Alcoholism } \\
\hline Yes & 12 & 4 & 0.029 \\
\hline No & 19 & 25 & \\
\hline \multicolumn{4}{|l|}{ Liver cirrhosis } \\
\hline Yes & 17 & 18 & 0.570 \\
\hline No & 14 & 11 & \\
\hline \multicolumn{4}{|l|}{$\operatorname{AFP}(n g / L)$} \\
\hline$<200$ & 21 & 19 & 0.855 \\
\hline$\geq 200$ & 10 & 10 & \\
\hline \multicolumn{4}{|l|}{$\operatorname{ALT}(\mathrm{U} / \mathrm{L})$} \\
\hline$<60$ & 19 & 23 & 0.128 \\
\hline$\geq 60$ & 12 & 6 & \\
\hline \multicolumn{4}{|l|}{ AST (U/L) } \\
\hline$<40$ & 18 & 22 & 0.144 \\
\hline$\geq 40$ & 13 & 7 & \\
\hline \multicolumn{4}{|l|}{ Tumor number } \\
\hline Single & 21 & 15 & 0.206 \\
\hline Multiple & 10 & 14 & \\
\hline \multicolumn{4}{|l|}{ Tumor size } \\
\hline$<5 \mathrm{~cm}$ & 16 & 15 & 0.993 \\
\hline$\geq 5 \mathrm{~cm}$ & 15 & 14 & \\
\hline \multicolumn{4}{|c|}{ Portal vein invasion } \\
\hline Yes & 5 & 8 & 0.282 \\
\hline No & 26 & 21 & \\
\hline \multicolumn{4}{|l|}{ TNM stage } \\
\hline I+II stage & 25 & 27 & 0.156 \\
\hline III+IV stage & 6 & 2 & \\
\hline
\end{tabular}

\section{DPT overexpression inhibits HCC cell growth in vitro and tumorigenicity in vivo}

We next assessed the levels of DPT in four independent HCC cell lines by Western blotting analysis. Figure 2A shows that DPT expression was downregulated in all HCC cells assessed. DPT overexpression experiments were next performed in Huh7 and HCCLM3 cells through the exogenous expression of DPT overexpression vectors (Figure 2B). Following DPT overexpression, cell growth and viability significantly declined in Huh7 and HCCLM3 cells vs. control groups (Figure $2 \mathrm{C}-\mathrm{D}, P<0.05$ ). We next injected $1 \times 10^{6}$ cells into nude mice and monitored mouse weights and tumor growth (Figure 2E). We found that HCCLM3-DPT xenografts were of reduced weight and size compared to control groups (Figure $2 \mathrm{~F}-\mathrm{G}, P<0.05)$. These data indicated that DPT overexpression inhibits HCC cell growth in vitro and HCC tumor formation in vivo. To sum up, this highlighted DPT as a potential therapeutic target in HCC. 


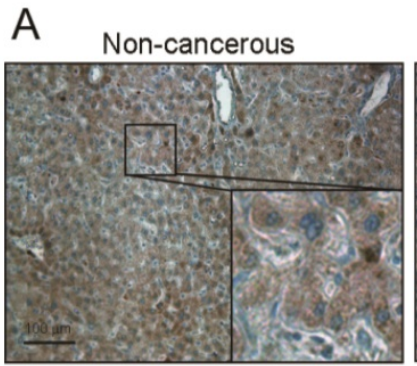

C

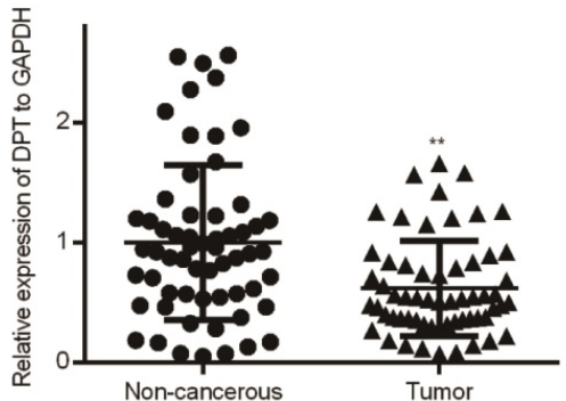

B

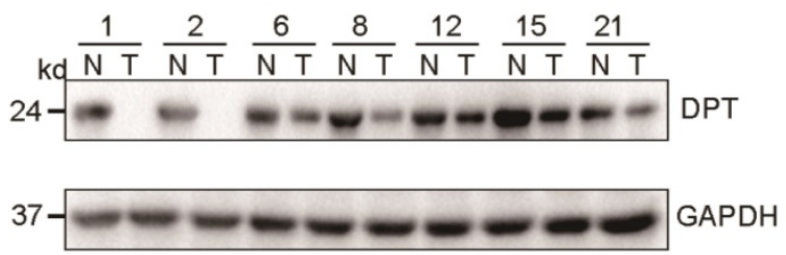

D

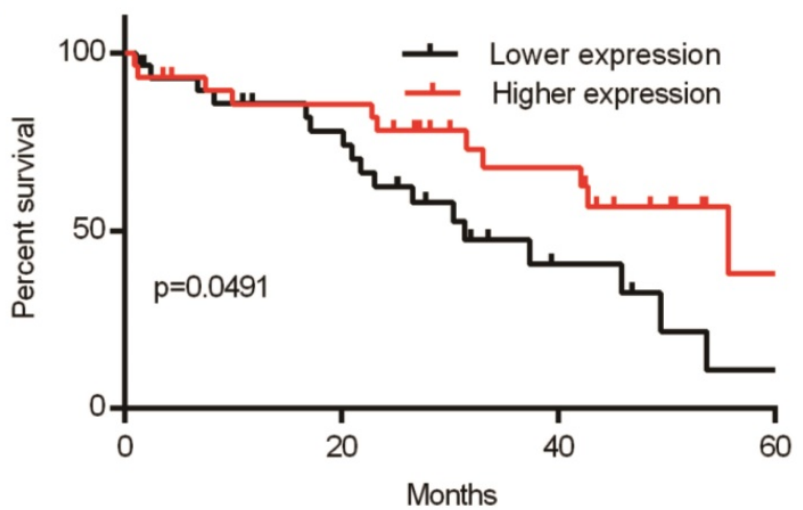

Figure 1. DPT levels in HCC tissue. (A) IHC analysis. (B) Representative Western blotting and (C) qPCR analysis of DPT levels in HCC vs. non-cancerous tissue. (D) Analysis of overall survival of patients with different DPT expression. All data are shown relative to GAPDH expression and were compared using a Student's $t$ test.

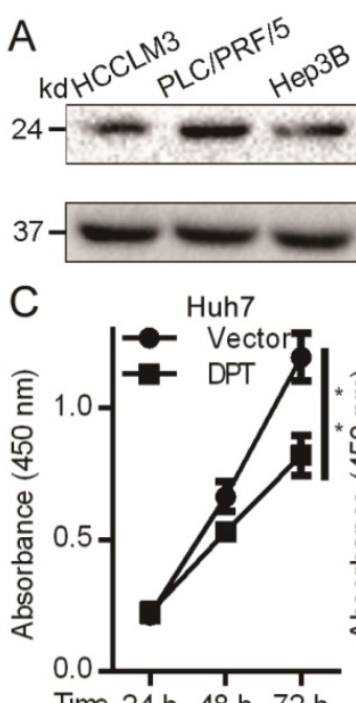

Time $24 \mathrm{~h} 48 \mathrm{~h} 72 \mathrm{~h}$

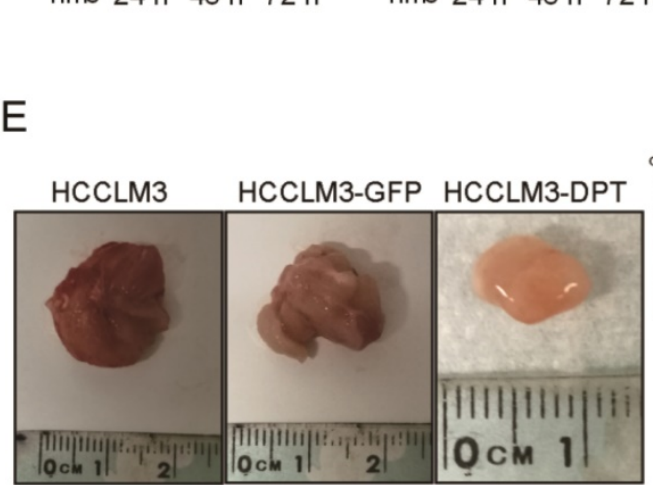

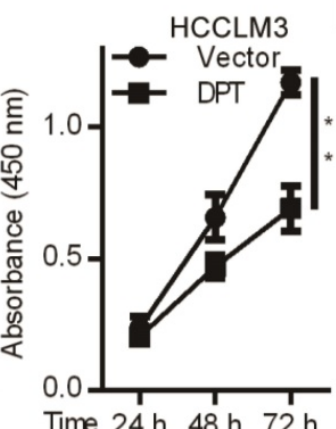

GAPDH
B

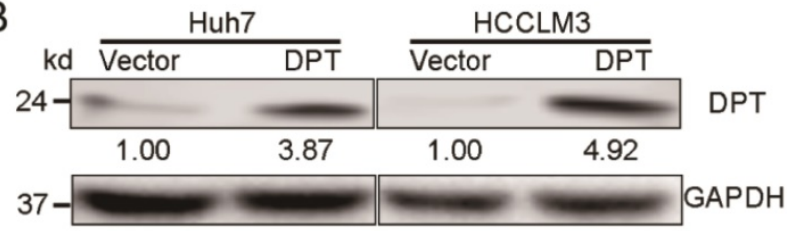

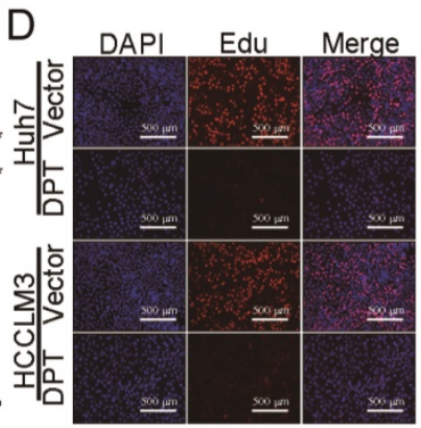

$\mathrm{F}$
Huh7

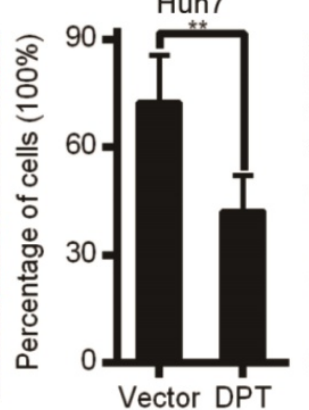

HCCLM3
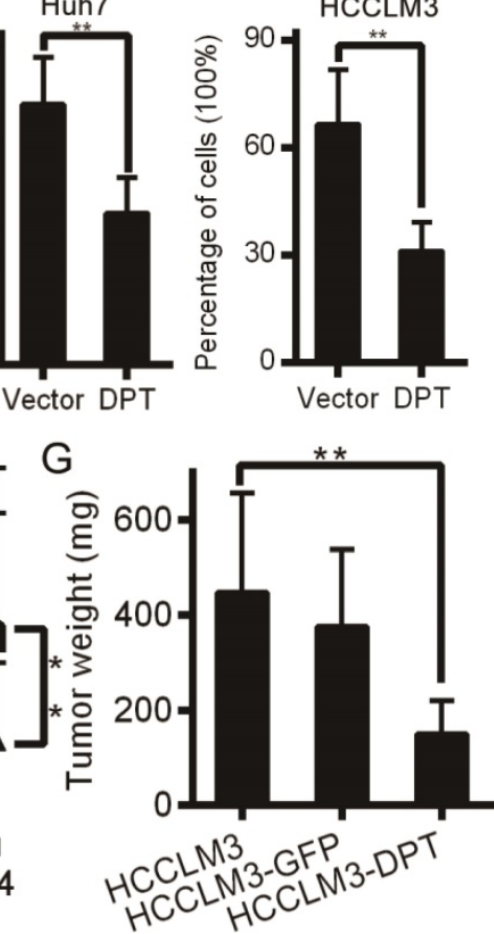

Figure 2. DPT inhibits HCC cell growth and tumorigenicity. (A) DPT levels in the indicated HCC cell lines. (B) DPT overexpression assessed by Western blotting. (C) Cell growth and MTT assays in response to exogenous DPT overexpression. (D) HCC cell lines were treated with DPT for $48 \mathrm{~h}$, EdU treated and fixed. The incorporation of EdU was assayed using a BeyoClick Kit. Representative images are shown. (E-G) Tumor growth curves and weights in response to exogenous DPT expression. Data were analyzed using a Student's t test. Data are the mean \pm SD. $* * P<0.01$. 

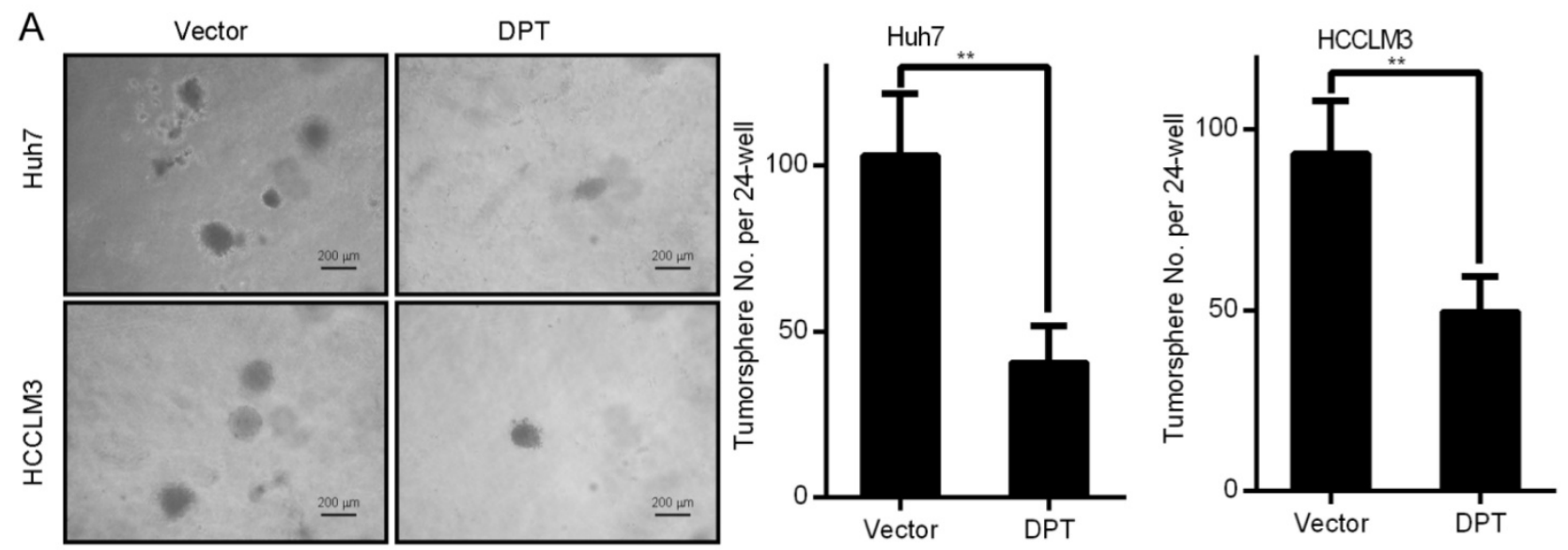

B

C
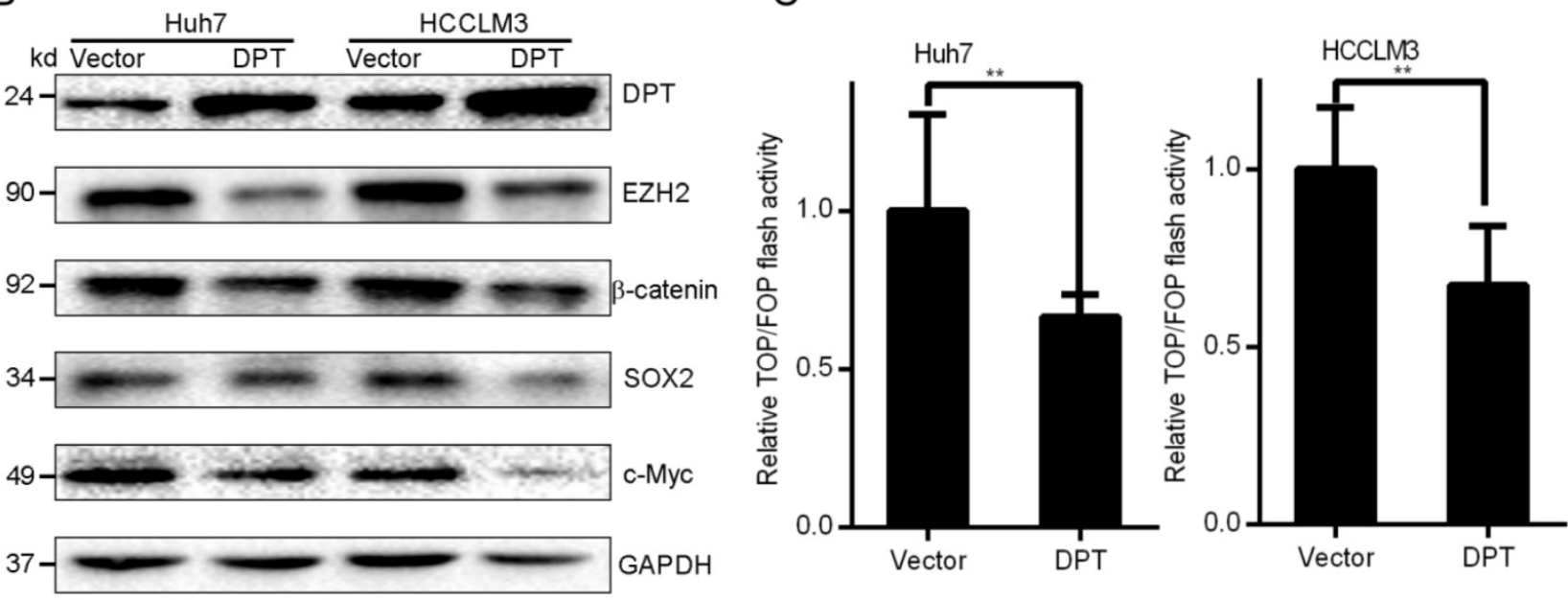

Figure 3. Exogenous DPT expression inhibits the CSC phenotypes of HCC. (A) Representative images of the tumor spheres in response to DPT overexpression. (B) Western blotting analysis. (C) Huh7 and HCCLM3 cells overexpressing DPT were transfected with TOP/FOPFlash reporter plasmids. Luciferase activity was assessed after 24 $\mathrm{h}$ of transfection. Data are the mean \pm SD of triplicate experiments. Data were compared through a Student's $t$ test. $* * P<0.01$.

Table 3. Spearman analysis of correlation between DPT and clinicopathological

\begin{tabular}{lll}
\hline Variables & \multicolumn{2}{l}{ DPT expression level } \\
\cline { 2 - 3 } & Spearman correlation & $p$ value \\
\hline Age (years, $<50$ vs. $\geq 50)$ & -0.255 & 0.049 \\
Gender (male/female) & 0.059 & 0.655 \\
Alcoholism (yes $/$ no) & -0.282 & 0.029 \\
Liver cirrhosis (yes/no) & 0.073 & 0.578 \\
AFP (ng/L, $<200$ vs. $\geq 200)$ & 0.024 & 0.858 \\
ALT $(\mathrm{U} / \mathrm{L},<60$ vs. $\geq 60$ ) & -0.197 & 0.132 \\
AST (U/L, $<40$ vs. $\geq 40$ ) & -0.189 & 0.149 \\
Tumor number (single/multiple) & 0.163 & 0.212 \\
Tumor size (cm, $<5$ vs. $\geq 5)$ & -0.001 & 0.993 \\
Portal vein invasion $($ yes $/$ no) & 0.139 & 0.290 \\
TNM stage (I+II vs III+IV) & -0.183 & 0.161
\end{tabular}

Bold numbers indicate significant differences $(P<0.05)$. HCC, hepatocellular carcinoma; AFP, a-fetoprotein; ALT, alanine aminotransferase; AST, aspartate aminotransferase; TNM, tumor node metastasis.

\section{DPT overexpression in HCC cells displays decreased characteristics of cancer stem cells (CSCs)}

Small populations of CSCs drive HCC tumor growth. CSCs undergo self-renewal in vitro. Cells exogenously expressing DPT or control cells were cultured in serum-free medium to permit the growth of tumor spheres (Figure 3A). In DPT overexpressing cells, colony formation in soft agar was found to be inhibited vs. control groups (Huh7 and HCCLM3). These data indicated that DPT inhibited the self-renewal capacity of HCC cells. Stem cells-related transcription factors are key to self-renewal capacity. In DPT overexpressing cells, the levels of SOX-2, $\mathrm{EZH} 2$, and c-Myc were significantly suppressed (Figure 3B) in addition to a loss of Wnt/ $\beta$-catenin signaling components evidenced through the levels of TOP-Flash reporter activity in Huh7 and HCCLM3 cells vs. control groups (Figure 3C, $P<0.15$ ) [9]. The culmination of these data highlight that the overexpression of DPT in HCC cells leads to a loss of CSC characteristics.

\section{DPT overexpression inhibits $W n t / \beta$-catenin signaling}

The effects of DPT overexpression were next assessed by Western blotting and immunofluorescent 
analysis. We observed a loss of $\beta$-catenin expression and nuclear accumulation (Figure 4A-B) in cells overexpressing DPT. To provide mechanistic insight into these effects, we analyzed the gene expression profiles of HCCLM3 cells overexpressing DPT (HCCLM3-DPT) vs. mock cells (HCCLM3-vector) with a focus on Wnt-related genes, assessed through commercially available qPCR arrays assay.

We found that DPT treatment led to the upregulation of 9 key Wnt-related genes, including WNT5A, WNT16, CXXC4, WNT11, CCND2, TCF7, WNT2B, RHOU and PPARD ( $\geq 2$-fold increase, Figure 4C, Table S1). The expression of EP300, BCL9, WNT8A, PORCN, PYGO1, WNT10A, MYC, WNT7A and CSNK1A1 decreased in response to DPT (Figure 4C, Table S1). Of the upregulated genes, CXXC4 (CXXC finger protein 4) was validated by Western blotting (Figure 4D). The expression of p27 also increased in response to DPT, whilst the expression of Cyclin D1 and $\beta$-catenin declined. These data suggest that DPT acts as a tumor suppressor in HCC through
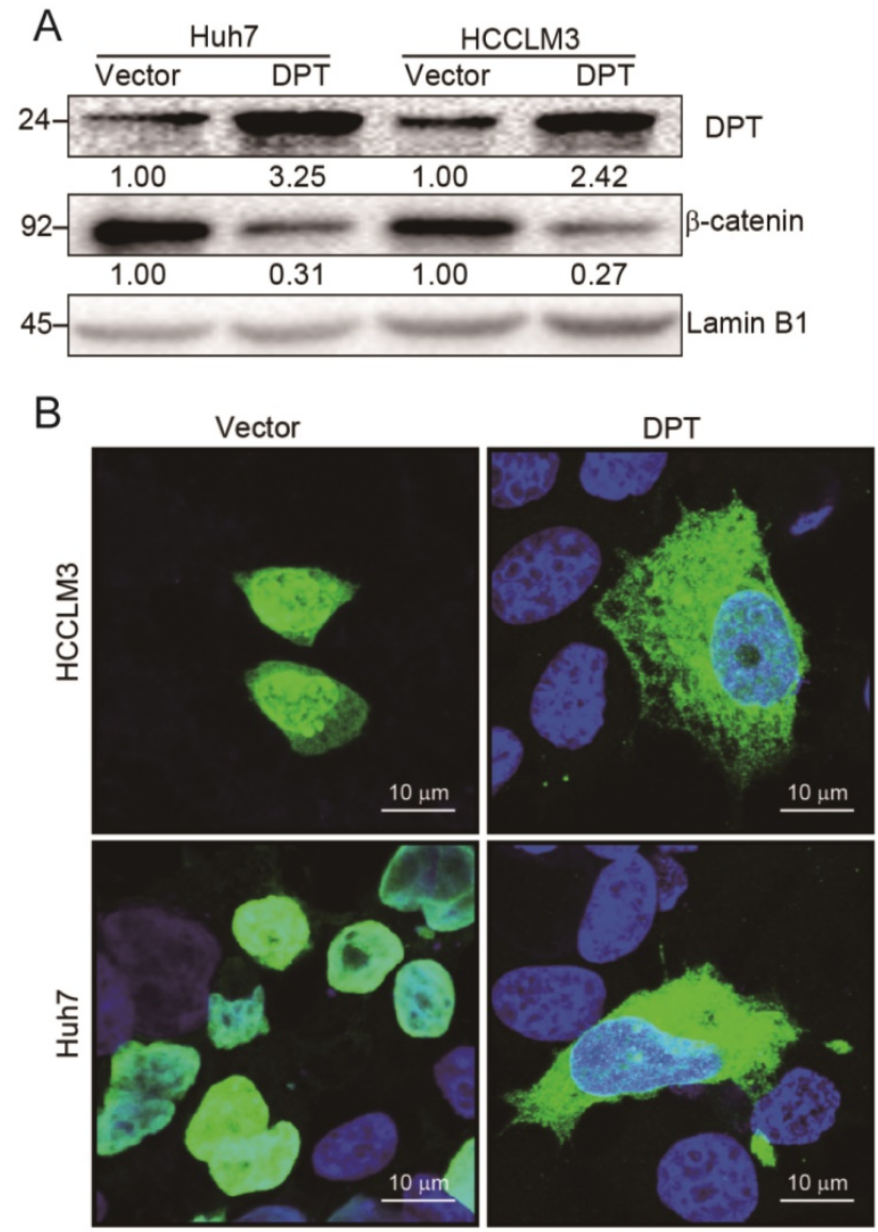

the inactivation of pro-oncogenic Wnt/ $\beta$-catenin signaling pathway.

\section{CXXC4 functions as a tumor suppressor gene in $\mathrm{HCC}$}

We next explored the tumor functions of CXXC4 in HCC. Boxplots illustrating the expression of CXXC4 in normal vs. HCC tissue were produced through MERAV analysis (Figure 5A). The median expression of CXXC4 was lower in healthy vs. HCC tissues. CXXC4 overexpression also led to a loss of Huh7 and HCCLM3 cell growth as assessed by MTT viability assays (Figure 5B). To further examine the effects of CXXC4 on HCC cells, both cell-types were engineered to overexpress CXXC4 through soft agar assays (Figure 5C). In response to CXXC4 overexpression, the growth of all assessed HCC cell lines was inhibited (Figure 5C). These data demonstrated that CXXC4 was an important tumor suppressor in HCC.
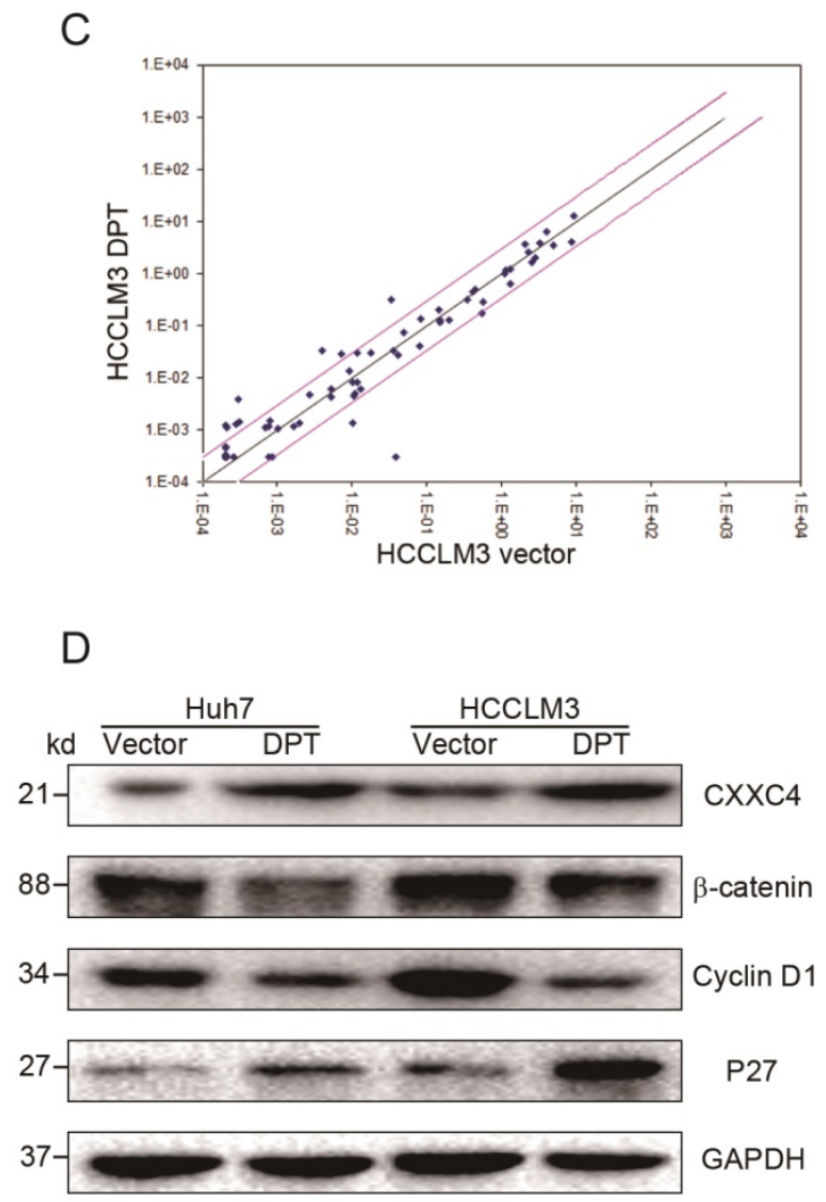

Figure 4. Overexpression of DPT inhibits $\mathbf{W n t} / \boldsymbol{\beta}$-catenin signaling. (A) $\beta$-catenin expression in the nucleus in response to DPT-overexpression. (B) Immunofluorescence analysis of $\beta$-catenin levels in DPT-overexpressing HCC cells. Cells were fixed in $4 \%$ PFA, treated with pepsin, and dehydrated using a gradient ethanol series. Cells were permeabilized in Triton X-100, blocked in PBS plus goat serum, and probed with anti- $\beta$ catenin antibodies and the appropriate secondary antibodies. Cell nuclei were counter-stained with DAPI. Representative confocal images were shown. (C) Expression of Wnt-related genes. Black line: fold-change in gene expression of 1. Pink line: desired fold change in gene expression threshold. (D) Expression of CXXC4, $\beta$-catenin, Cyclin D1 and P27 assessed by Western blotting. Data are the mean \pm SD and were compared using a student's t-test. $* P<0.05$. 
A
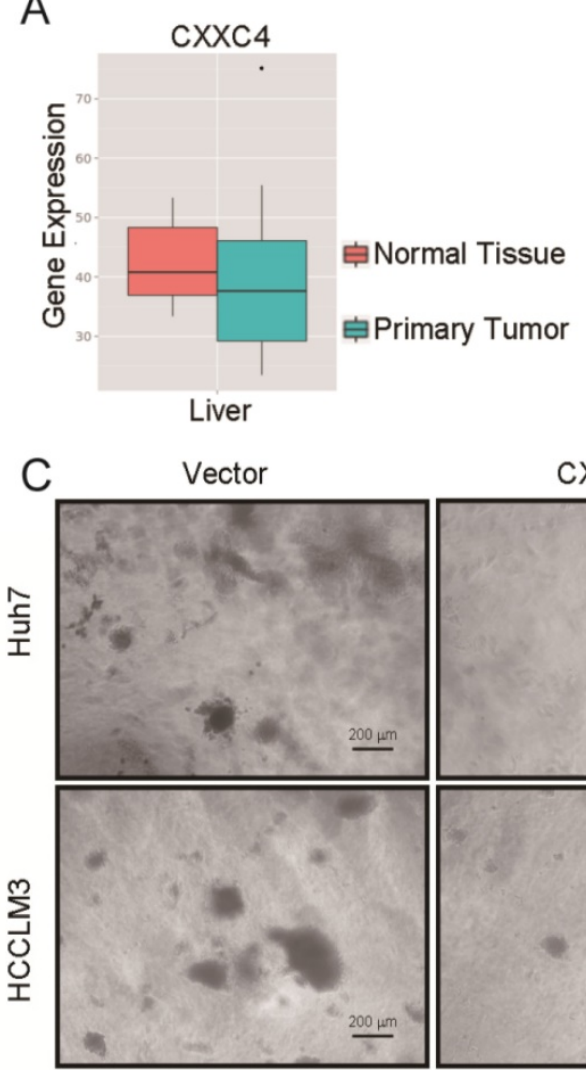

B
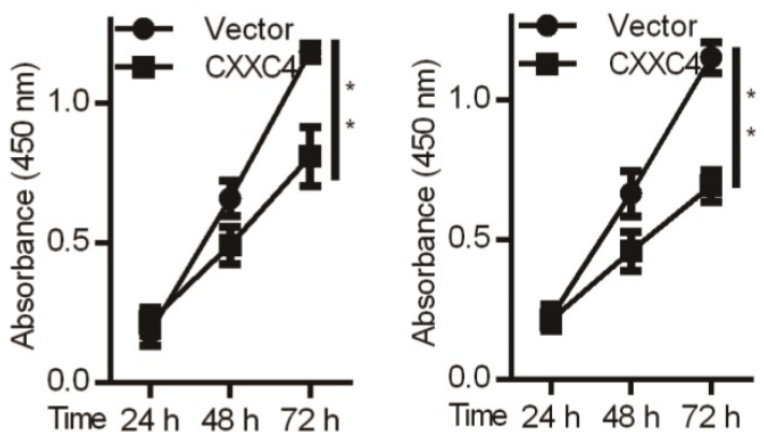

CXXC4
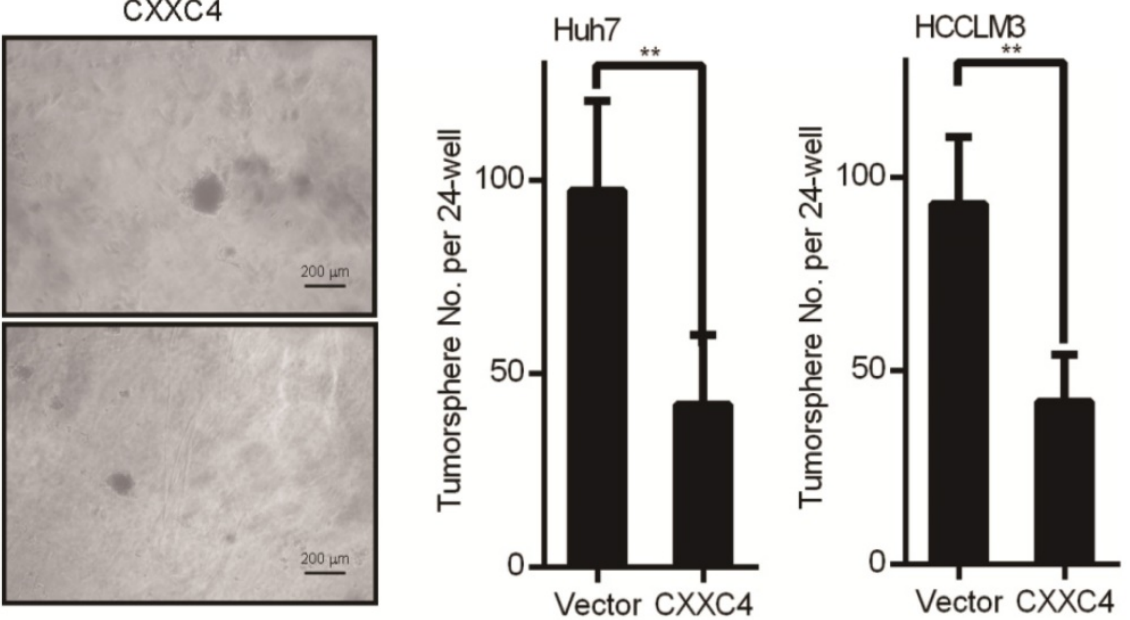

Figure 5. CXXC4 inhibits HCC cell proliferation and colony formation. (A) Metabolic gEne RApid Visualizer (MERAV) boxplots for CXXC4 expression in normal vs. HCC tissue. (B) MTT assays in cells overexpressing CXXC4. Values: mean \pm SD of absorbance at $450 \mathrm{~nm}(\mathrm{n}=5)$. (C) Colony formation assays. Data are the mean \pm SD $(\mathrm{n}=$ 3); $* p<0.05, * * p<0.01$.

A

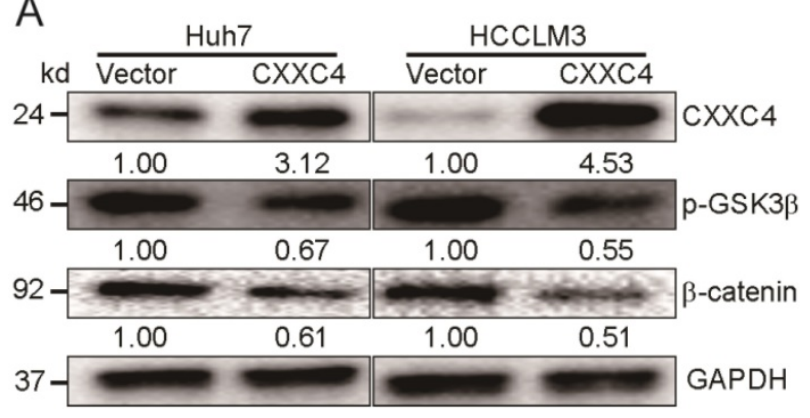

B

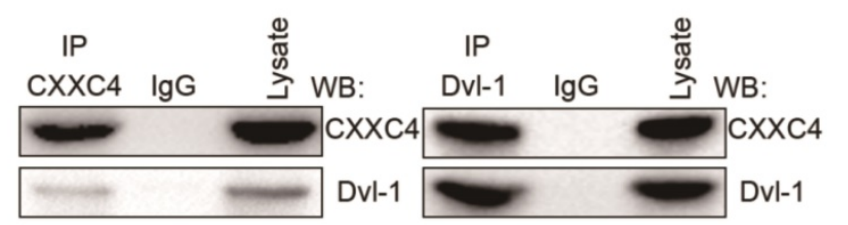

Figure 6. CXXC4 inhibits Wnt signaling in HCC cells. (A) Levels of $\beta$-catenin and p-GSK-3 $\beta$ in Huh7 cells in response to CXXC4 overexpression assessed by western blotting. (B) Cells $\left(2 \times 10^{6}\right)$ were lysed in Co-IP buffer and labeled with anti-flag or anti-Dvl-1 antibodies. Protein A/G conjugated to agarose was then added for $2 \mathrm{~h}$ and pelleted through centrifugation. Control samples contained rabbit lgG alone. Beads were washed in IP buffer and mixed with $2 \times$ loading buffer. Samples were boiled for 5 min and assessed by Western blotting analysis. Representative IPs showing the Dvl-1 interaction with CXXC4 are shown.

\section{CXXC4 inhibits Wnt signaling in HCC}

CXXC4 is a negative regulator of $\mathrm{Wnt} / \beta$-catenin signaling in renal cell carcinoma (RCC) and gastric cancer in the previously report [10]. We assessed the contribution of CXXC4 to Wnt signaling in HCC. Exogenous CXXC4 expression reduced $\beta$-catenin expression (Figure 6A) and p-GSK-3 $\beta$ levels (Figure 6A). In this study, we observed a CXXC4 and Dvl-1 interaction through Co-IP assays in HCCLM3 cells (Figure 6B) confirming this mechanism in HCC. This furthers our understanding of the molecular mechanisms governing HCC progression.

\section{DPT inactivates Wnt signaling through upregulating $\mathrm{CXXC4}$}

We next assessed whether DPT inhibits Wnt signaling and HCC progression through its effects on CXXC4. We found that the downregulation of DPT enhanced $\beta$-catenin expression in HCCLM3 cells (Figure 7A), whilst the silencing of CXXC4 induced $\beta$-catenin levels (Figure 7A). We also found that the inhibitory effects of DPT shRNA could be reversed by the overexpression of CXXC4 (Figure 7B), suggesting 
that DPT inactivates Wnt signaling to promote HCC carcinogenesis. Cell growth and Wnt were inhibited by the exogenous expression of CXXC4 (Figure 7B-C). CXXC4 shRNA cells were able to maintain their inhibition of cell growth and $\beta$-catenin upon simultaneous CXXC4 activation (Figure 7A-7C). The culmination of these data confirmed that DPT inhibits Wnt signaling in HCC through its ability to upregulation of CXXC4 expression (Figure 7D).

\section{Discussion}

HCC is now the $2^{\text {nd }}$ leading cause of cancer associated death globally and a major cause of mortality in China and across the world [11]. Whilst anti-HCC therapies have improved and evolved, the 5 -year overall survival rates in HCC patients remain dismal, due to the high levels of recurrence and metastasis following surgery $[12,13]$. Therefore, an improved understanding of HCC progression and metastasis is therefore required to improve HCC therapy.

It has been reported that DPT competes with decorin for its interaction with TGF- $\beta$ [3], which regulates a range of pathological processes, including tumor progression, growth and survival [14]. We found that the overexpression of DPT suppresses
HCC cell proliferation. However, the effects of DPT on HCC cell growth and its relationship to Wnt signaling is undefined. Emerging evidence suggests the existence of a cross-talk mechanism between TGF- $\beta$, integrins and the ECM [15]. Further assessments of the contribution of DPT to this signaling axis will enhance our understanding of the mechanisms of HCC progression.

Canonical Wnt signaling regulates HCC cell self-renewal and drug-resistance, widely contributing to the poor prognosis of HCC $[16,17]$. Despite the incomplete statistics, approximately $30 \%$ of HCC cases display aberrant Wnt activation [18]. The dysregulation of the Wnt/ $\beta$-catenin axis is a known oncogenic driver, and represents an attractive therapeutic strategy for HCC [19]. DPT regulates the progression and development of several human cancers $[2,6,20]$. DPT is downregulated during cancer malignancy, as shown by Yamatoji and colleagues in human OSCC. Guo and coworkers additionally reported that DPT expression is downregulated in papillary thyroid cancer [21]. In this study, using Western blotting analysis, bioinformatics, qPCR and IHC assessments, DPT expression was shown for the first time to be suppressed in HCC.

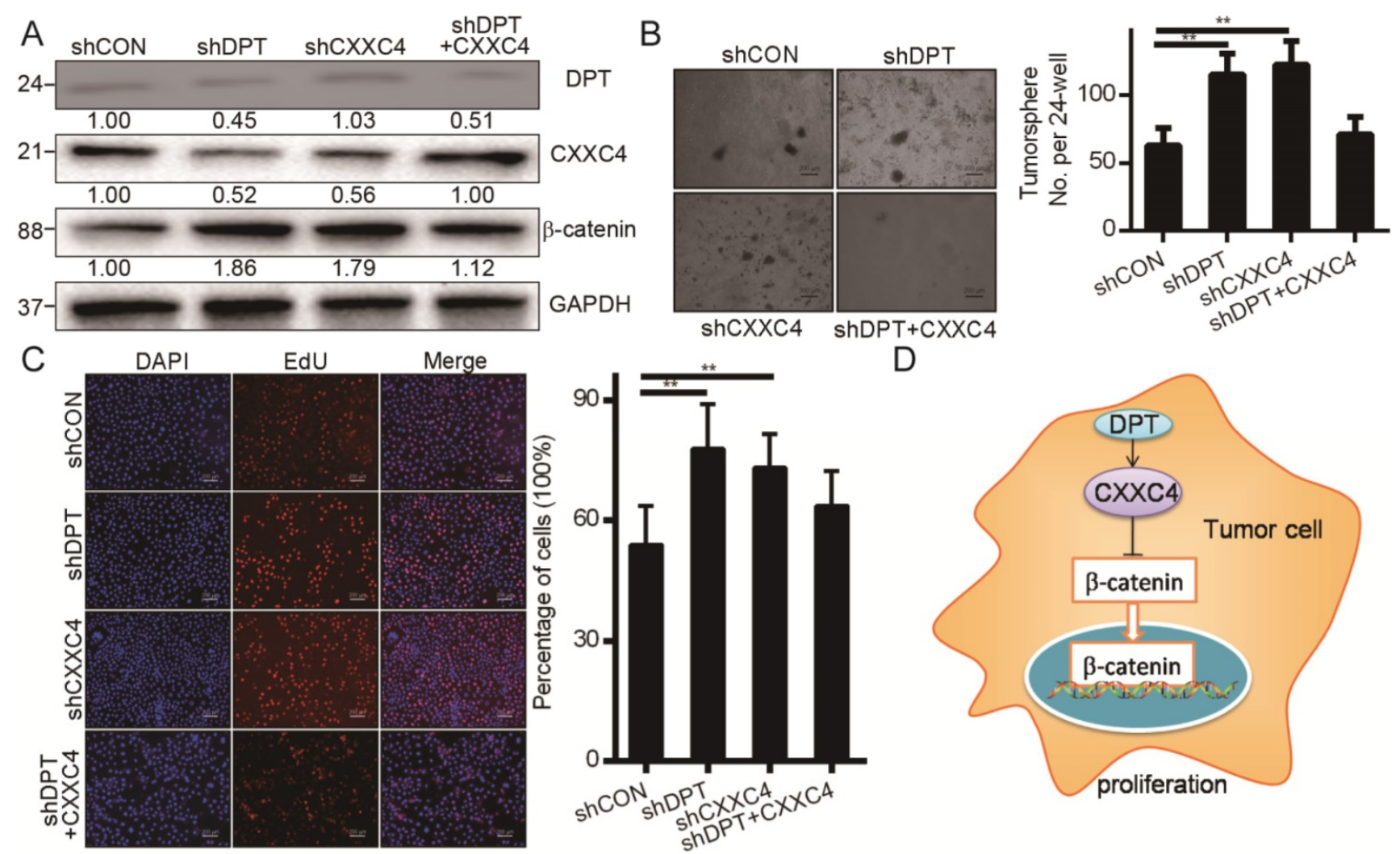

Figure 7. CXXC4 inactivates Wnt through CXXC4. (A) CXXC4 and $\beta$-catenin levels in shDPT, shCXXC4 or shDPT cells assessed through Western blotting analysis. (B) Effect of shDPT, shCXXC4 or shDPT and CXXC4 on colony formation. (C) Huh7 cells were treated with shDPT, shCXXC4 or shDPT and CXXC4 for $24 \mathrm{~h}$ and stained with EdU and DAPI. (D) Proposed model: Indicating that DPT upregulates the expression of CXXC4. CXXC4 could stabilize the degradation of $\beta$-catenin and reduce $\beta$-catenin expression. 
Aberrant Wnt/ $\beta$-catenin signaling due to genetic changes in APC or CTNNB1 is a driver of an array of human malignancies [22, 23]. However, aberrant cellular CTNNB1 translocation occurs in up to $90 \%$ of HCC cases but CTNNB1 mutations only occur in $2.8 \%-44 \%$ of cases, suggesting other mechanisms of Wnt deregulation in HCC [24]. The tumor suppressor functions of CXXC4 are also well-characterized and CXXC4 inhibits Wnt/ $\beta$-catenin signaling through its interaction with Disheveled [25-27]. Aberrant Wnt signaling pathway activation predicts poor cancer patient prognosis [28]. EZH2 performs a pivotal and essential function in maintaining cancer properties in Wnt/ $\beta$-catenin signaling activation [29]. SOX2 has a crucial role in the proliferation of cancer Cells through c-MYC in Wnt/ $\beta$-catenin pathway [30]. In this study, we identified DPT as a mechanism to inactivate Wnt signaling through CXXC4, representing a novel epigenetic mechanism of HCC mediated Wnt signaling (Figure 7D).

In summary, our data provide the first evidence of the epigenetic upregulation of DPT in HCC and its effects on Wnt signaling. We show that the DPT mediated repression of CXXC4 leads to the inhibition of constitutively active Wnt/ $\beta$-catenin, consistent with studies highlighting the ability of EZH2 to promote carcinogenesis through the repression of ADRB2, CDH1, PSP94 and DAB2IP and other tumor suppressors [25]. These data highlight how epigenetic regulation in addition to genetic changes regulates the aberrant activation of $\mathrm{Wnt} / \beta$-catenin signaling in HCC.

In summary, we have revealed CXXC4 as a target for DPT and its suppression during HCC development. We show that DPT functions as a tumor suppressor in HCC and inhibits Wnt signaling through CXXC4 mediated $\beta$-catenin degradation.

\section{Conclusions}

Here, we reveal that DPT is poorly expressed in HCC tissues and cells, and that its recovery leads to anti-HCC effects. Mechanistically, we reveal that DPT suppresses Wnt signaling through its activation of CXXC4, regulating downstream targets including EZH2, $\beta$-catenin, SOX2 and c-Myc. These findings highlight the anti-HCC effects of DPT revealing its potential as a novel therapeutic target for HCC.

\section{Supplementary Material}

Supplementary figures and tables. http://www.jcancer.org/v11p6288s1.pdf

\section{Acknowledgements}

We would like to thank Dr. Ning Ma (Stanford University, CA) for their kind gift of reagents. We also would like to thank Dr. Bianca Troncarelli Flores (Stanford University, CA) for critical reading of the manuscript.

\section{Funding}

This work was supported by National Natural Science Foundation of China (Grant No. 81402579), the Key Program of Research and Development Foundation of Shandong province (Grant No. 2017GSF18179), Source Innovation Foundation of Qingdao (Grant No. 18-2-2-79-jch) and "Clinical Medicine $+X^{\prime \prime}$ of Qingdao University (Grant No. CMX201729). The funders had no role in study design, data collection and analysis, decision to publish, or preparation of the manuscript.

\section{Ethics approval and consent}

This work has been approved by the ethical committees at the Affiliated Hospital of Qingdao University, and we have obtained written informed consent from all participants involved in the study. Ethics approval for animal research: applicable.

\section{Competing Interests}

The authors have declared that no competing interest exists.

\section{References}

1. Choo SP, Tan WL, Goh BKP, Tai WM, Zhu AX. Comparison of hepatocellular carcinoma in Eastern versus Western populations. Cancer. 2016; 122: 3430-46.

2. Okamoto O, Fujiwara S. Dermatopontin, a novel player in the biology of the extracellular matrix. Connective tissue research. 2006; 47: 177-89.

3. Okamoto O, Fujiwara S, Abe M, Sato Y. Dermatopontin interacts with transforming growth factor beta and enhances its biological activity. The Biochemical journal. 1999; 337 ( Pt 3): 537-41.

4. Takeda U, Utani A, Wu J, Adachi E, Koseki H, Taniguchi M, et al. Targeted disruption of dermatopontin causes abnormal collagen fibrillogenesis. The Journal of investigative dermatology. 2002; 119: 678-83.

5. Sidgwick GP, Bayat A. Extracellular matrix molecules implicated in hypertrophic and keloid scarring. Journal of the European Academy of Dermatology and Venereology : JEADV. 2012; 26: 141-52.

6. Yamatoji M, Kasamatsu A, Kouzu Y, Koike H, Sakamoto Y, Ogawara K, et al. Dermatopontin: a potential predictor for metastasis of human oral cancer. International journal of cancer. 2012; 130: 2903-11.

7. Liu S, Qiu J, He G, Liang Y, Wang L, Liu C, et al. LncRNA MALAT1 acts as a miR-125a-3p sponge to regulate FOXM1 expression and promote hepatocellular carcinoma progression. J Cancer. 2019; 10: 6649-59.

8. Jin $\mathrm{H}$, Sperka $\mathrm{T}$, Herrlich $\mathrm{P}$, Morrison $\mathrm{H}$. Tumorigenic transformation by CPI-17 through inhibition of a merlin phosphatase. Nature. 2006; 442: 576-9.

9. Chen X, Huan H, Liu C, Luo Y, Shen J, Zhuo Y, et al. Deacetylation of beta-catenin by SIRT1 regulates self-renewal and oncogenesis of liver cancer stem cells. Cancer letters. 2019; 463: 1-10.

10. Kojima T, Shimazui T, Hinotsu S, Joraku A, Oikawa T, Kawai K, et al. Decreased expression of CXXC4 promotes a malignant phenotype in renal cell carcinoma by activating Wnt signaling. Oncogene. 2009; 28: 297-305.

11. Thomas MB, Jaffe D, Choti MM, Belghiti J, Curley S, Fong Y, et al. Hepatocellular carcinoma: consensus recommendations of the National Cancer Institute Clinical Trials Planning Meeting. Journal of clinical oncology : official journal of the American Society of Clinical Oncology. 2010; 28: 3994-4005.

12. Aldrighetti L, Pulitano C, Catena M, Arru M, Guzzetti E, Halliday J, et al. Liver resection with portal vein thrombectomy for hepatocellular carcinoma with vascular invasion. Annals of surgical oncology. 2009; 16: 1254

13. Yang PC, Ho CM, Hu RH, Ho MC, Wu YM, Lee PH. Prophylactic liver transplantation for high-risk recurrent hepatocellular carcinoma. World journal of hepatology. 2016; 8: 1309-17.

14. Derynck R, Akhurst RJ, Balmain A. TGF-beta signaling in tumor suppression and cancer progression. Nature genetics. 2001; 29: 117-29. 
15. Munger JS, Sheppard D. Cross talk among TGF-beta signaling pathways, integrins, and the extracellular matrix. Cold Spring Harbor perspectives in biology. 2011; 3: a005017.

16. Li J, Yu B, Deng P, Cheng Y, Yu Y, Kevork K, et al. KDM3 epigenetically controls tumorigenic potentials of human colorectal cancer stem cells through Wnt/beta-catenin signalling. Nature communications. 2017; 8: 15146.

17. van de Wetering M, Sancho E, Verweij C, de Lau W, Oving I, Hurlstone A, et al. The beta-catenin/TCF-4 complex imposes a crypt progenitor phenotype on colorectal cancer cells. Cell. 2002; 111: 241-50.

18. Guichard C, Amaddeo G, Imbeaud S, Ladeiro Y, Pelletier L, Maad IB, et al. Integrated analysis of somatic mutations and focal copy-number changes identifies key genes and pathways in hepatocellular carcinoma. Nature genetics. 2012; 44: 694-8.

19. Song J, Xie C, Jiang L, Wu G, Zhu J, Zhang S, et al. Transcription factor AP-4 promotes tumorigenic capability and activates the Wnt/beta-catenin pathway in hepatocellular carcinoma. Theranostics. 2018; 8: 3571-83.

20. Fu Y, Feng MX, Yu J, Ma MZ, Liu XJ, Li J, et al. DNA methylation-mediated silencing of matricellular protein dermatopontin promotes hepatocellular carcinoma metastasis by alpha3beta1 integrin-Rho GTPase signaling. Oncotarget. 2014; 5: 6701-15.

21. Guo Y, Li H, Guan H, Ke W, Liang W, Xiao H, et al. Dermatopontin inhibits papillary thyroid cancer cell proliferation through MYC repression. Molecular and cellular endocrinology. 2019; 480: 122-32.

22. Barker N, Clevers H. Mining the Wnt pathway for cancer therapeutics. Nature reviews Drug discovery. 2006; 5: 997-1014.

23. Li ZQ, Ding W, Sun SJ, Li J, Pan J, Zhao C, et al. Cyr61/CCN1 is regulated by Wnt/beta-catenin signaling and plays an important role in the progression of hepatocellular carcinoma. PloS one. 2012; 7: e35754.

24. Perugorria MJ, Olaizola P, Labiano I, Esparza-Baquer A, Marzioni M, Marin JJG, et al. Wnt-beta-catenin signalling in liver development, health and disease. Nature reviews Gastroenterology \& hepatology. 2019; 16: 121-36.

25. Lu H, Sun J, Wang F, Feng L, Ma Y, Shen Q, et al. Enhancer of zeste homolog 2 activates wnt signaling through downregulating CXXC finger protein 4. Cell death \& disease. 2013; 4: e776.

26. Lu H, Jin W, Sun J, Feng L, Lan H, Shen Q, et al. New tumor suppressor CXXC finger protein 4 inactivates mitogen activated protein kinase signaling. FEBS letters. 2014; 588: 3322-6.

27. Hino S, Kishida S, Michiue T, Fukui A, Sakamoto I, Takada S, et al. Inhibition of the Wnt signaling pathway by Idax, a novel Dvl-binding protein. Molecular and cellular biology. 2001; 21: 330-42.

28. de Sousa EMF, Colak S, Buikhuisen J, Koster J, Cameron K, de Jong JH, et al. Methylation of cancer-stem-cell-associated Wnt target genes predicts poor prognosis in colorectal cancer patients. Cell stem cell. 2011; 9: 476-85.

29. Chen JF, Luo X, Xiang LS, Li HT, Zha L, Li N, et al. EZH2 promotes colorectal cancer stem-like cell expansion by activating p21cip1-Wnt/beta-catenin signaling. Oncotarget. 2016; 7: 41540-58.

30. Park SB, Seo KW, So AY, Seo MS, Yu KR, Kang SK, et al. SOX2 has a crucial role in the lineage determination and proliferation of mesenchymal stem cells through Dickkopf-1 and c-MYC. Cell death and differentiation. 2012; 19: 534-45. 